\title{
Expression of ERCC1, TYMS, RRM1, TUBB3, non-muscle myosin II, myoglobin and MyoD1 in lung adenocarcinoma pleural effusions predicts survival in patients receiving platinum-based chemotherapy
}

\author{
HAIJIAO JIANG ${ }^{1}$, HE WANG $^{2}$, SHIYU WANG ${ }^{3}$, ZHENGTONG PEI ${ }^{2}$, ZHIMIN FU $^{1}$, CHANGQING FANG ${ }^{1}$, \\ JIAN WANG ${ }^{1}$, QINGJIE LU ${ }^{4}$, ENHUA WANG ${ }^{1}$ and JIANHUA LI ${ }^{1}$

\begin{abstract}
${ }^{1}$ Department of Pathology, College of Basic Medical Sciences and First Affiliated Hospital, China Medical University, Philadelphia, PA 19140; ${ }^{3}$ Department of Medicine, The Commonwealth Medical College, Scranton, PA 18905, USA; ${ }^{4}$ Department of Pathology, Second Affiliated Hospital of China Medical University, Shenyang, Liaoning 110011, P.R. China
\end{abstract} \\ Shenyang, Liaoning 110013, P.R. China; ${ }^{2}$ Department of Pathology and Lab Medicine, Temple University School of Medicine,
}

Received December 14, 2013; Accepted October 24, 2014

DOI: $10.3892 / \mathrm{mmr} .2014 .3141$

\begin{abstract}
The association between the expression of excision repair cross-complementing gene 1 (ERCC1), thymidylate synthase (TYMS), ribonuleotide reductase M1 (RRM1), $\beta I I I-t u b u l i n(T U B B 3)$, non-muscle myosin II, myoglobin and MyoD1 in metastatic lung adenocarcinoma, and clinical outcomes with platinum-based chemotherapy treatment is not well-established. Recently, increasing attention has been focused on the involvement of ERCC1, TYMS, RRM1 and TUBB3 in the development of drug resistance. There has been less research into the role of muscle myosin II, myoglobin and MyoD1 in the pathogenesis of lung cancer, although these genes are known to have important functions within tumor cells. In the current study, malignant pleural effusion from 116 patients with untreated lung adenocarcinoma diagnosed between 2011 and 2012, were collected. The protein expression levels of ERCC1, TYMS, RRM1 and TUBB3 were evaluated with immunocytochemistry and western blot analysis. The expression levels of non-muscle myosin II, myoglobin and MyoD1 were measured in a subset of 50 patients, treated with platinum-based chemotherapy. The association of each of these seven factors with one another, as well as with patient survival were analyzed. Immunohistochemistry demonstrated that the percentage of pleural fluid samples from patients with lung adenocarcinoma expressing ERCC1, TYMS, RRM1 and TUBB3 was
\end{abstract}

Correspondence to: Professor Jianhua Li, Department of Pathology, College of Basic Medical Sciences and First Affiliated Hospital, China Medical University, 77 Puhe Road, New District of Shenbei, Shenyang, Liaoning 110013, P.R China

E-mail: lijh@mail.cmu.edu.cn

Key words: drug resistance genes, lung carcinoma, survival
$37,36.2,82.7$ and $69.8 \%$, respectively. In the subset of 50 patients in whom the remaining factors were analyzed, the percentage expressing non-muscle myosin II was $48 \%$, for myoglobin the figure was $40 \%$ and for MyoD1 it was $38 \%$. There was a positive correlation between each pair of the above seven molecules with the exception of TYMS and RRM1. Expression of ERCC1, TYMS, TUBB3, non-muscle myosin II, myoglobin and MyoD1 genes was associated with decreased survival in patients with metastatic lung adenocarcinoma. Expression of ERCC1, TYMS, TUBB3, non-muscle myosin II, myoglobin and MyoD1 was also associated with decreased survival rates of patients with lung adenocarcinoma treated with platinum-based chemotherapy. These factors may be used as clinical biomarkers to predict the biological behavior and chemoresistance of tumor cells, and the survival of patients with lung carcinoma.

\section{Introduction}

Carcinoma of the lung is responsible for the highest rate of cancer-related mortality. The majority of these fatalities (84\%) are caused by non-small cell lung cancer (NSCLC) (1). Over half of the cases of NSCLC have detectable distant metastasis at the time of diagnosis, including $16 \%$ with pleural metastasis. They are thus classified as stage IIIB or IV $(2,3)$. Platinum-based chemotherapy is the treatment of choice for advanced and metastatic NSCLC. Although novel chemotherapeutic agents continue to emerge, the development of chemoresistance remains a significant problem (4). A number of mechanisms lead to chemoresistance; it has been shown that altered expression of even a single gene can result in cancer cell resistance to particular drugs (5). Recent studies have highlighted four genes involved in drug-resistance in NSCLC: Excision repair cross-complementing gene 1 (ERCC1); thymidylate synthase (TYMS); ribonucleotide reductase M1 (RRM1); and $\beta$ III-tubulin (TUBB3) (6). These molecules are important in DNA repair, and cell replication and division. 
ERCC1 is a key member of the nucleotide repair exonuclease family. The ERCC1 protein forms a heterodimer with XPF (ERCC4) and acts as a 5'-endonuclease to excise the lesion as well as nucleotides surrounding the damaged site, a process termed nucleotide excision repair (NER). ERCC1 is the rate-limiting enzyme in NER (7). A recent study in NSCLC cell lines showed that elevated DNA repair capacity, as a result of enhanced ERCC1 expression, induced drug resistance (8). Immunohistochemical studies have shown that in patients with NSCLC, only those individuals with ERCC1-negative tumors benefit from cisplatin-based chemotherapy $(9,10)$. TYMS is a rate-limiting enzyme in thymidylate synthesis and dTMP is a required precursor for nucleic acid synthesis (11). Pemetrexed is an antifolate agent that predominantly targets TYMS. Treatment with pemetrexed leads to thymidine and purine deficiency, thereby inhibiting DNA synthesis, cell replication and tumor growth. A number of studies have demonstrated that overexpression of TYMS correlates with resistance to pemetrexed $(12,13)$. $\mathrm{RR}$ is comprised of two regulatory subunits of RRM1 and two catalytic subunits of RRM2. RR is a rate-limiting enzyme for DNA synthesis and is the only enzyme that reduces ribonucleotides into deoxyribonucleotides. It is thus required for DNA synthesis and repair (14). Vilmar et al (15) showed that patients with RRM1-negative advanced NSCLC who were treated with cisplatin and vinorelbine, exhibited an improvement in disease control, longer progression-free survival (PFS) and longer overall survival (OS). Tubulin is a key constituent of the cytoskeleton, which participates in cell movement, intracellular transportation and cell mitosis. $\beta$-tubulin is the target of tubulin-binding agents. These therapies inhibit mitosis by interfering with mitotic spindle formation and are effective in treating a number of types of tumor. The most commonly used anti-microtubule drugs are paclitaxel, docetaxel and vincristine. Taxol promotes microtubule polymerization and increases the stability of polymerized microtubules. The persistent presence of microtubules during cell division induces apoptosis. TUBB3 is primarily expressed in the central and peripheral nervous system and is strongly expressed during embryonic development (16). Expression of TUBB3 in NSCLC has been associated with drug resistance to tubulin-binding agents (17).

Myosin II is multifunctional, it has been identified in muscle and non-muscle cells. Within muscle cells, myosin II provides the driving force for muscle contraction, whereas in non-muscle cells, it is an integral component of the cytoskeleton, participating in cell movement, cytoplasmic flow, organelle movement and mitosis. A recent study demonstrated that myosin II is involved in lysophosphatidic acid (LAP)-mediated breast cancer cell migration and invasion (18). Myoglobin is an oxygen-carrying globin, which is primarily distributed in skeletal muscle cells and cardiomyocytes. Myoglobin reversibly binds to and releases oxygen, thereby promoting oxygen diffusion from cytoplasm to mitochondria (19). Flonta et al (20) identified myoglobin expression in a variety of epithelial tumors, including lung carcinoma. However, no expression was detected in corresponding healthy tissues. Myoglobin has been proposed to be important for tumor survival in hypoxia, tumor cell metabolism and tumor growth. MyoDl is a transcription factor, which is a member of the myogenic determination family, MyoD. In breast cancer cells, MyoD binds to the E-box on the BRCA1 promoter and activates its transcription by inducing histone acetylation (21). During embryonic development, MyoD1 binds to the DNA E-box of stem cells, to induce myogenic differentiation (22). In routine pathological practice, $\mathrm{MyoDl}$ is primarily used as a marker for rhabdomyosarcoma.

In the current study, the expression of ERCC1, TYMS, RRM1, TUBB3, myosin II, myoglobin and MyoD1 in pleural effusions from patients with lung adenocarcinoma was evaluated and the correlation between expression of these factors was analyzed. Clinical follow-up was conducted in a subset of patients following platinum-based chemotherapy and the association between survival and protein expression was also investigated. The study aimed to provide novel insights into the molecular pathology of lung adenocarcinoma.

\section{Materials and methods}

Patient recruitment and cytological sample collection. Pleural fluid from 116 patients with untreated metastatic lung adenocarcinoma was collected between January 2011 and July 2012 at the China Medical University First Affiliated Hospital (Shenyang, China). Diagnoses were made cytologically. This procedure included the preliminary diagnosis of suspicious tumor cells by microscopy, and the subsequent hematoxylin and eosin staining and immunocytochemical staining of epithelial tumor markers, in order to make a comprehensive assessment. Cytological samples from 20 patients with inflammation-induced pleural effusions were collected as the control group. The 116 patients with malignant pleural effusions were comprised of 47 males and 69 females, with a mean age of 57 years (range, 26-87 years). Clinical follow-up data were available in 48 patients with metastatic lung adenocarcinoma, treated with platinum-based chemotherapy. The follow-up periods varied between 15 and 28 months (until patients succumbed to disease or 30th April, 2013).

This study was approved by the ethics committee of China Medical University (Shenyang, China).

ThinPrep ${ }^{\circledR}$ cytology test (TCT) and cell block preparation. Freshly collected pleural effusions $(25 \mathrm{ml})$ were centrifuged at $670 \mathrm{x} \mathrm{g}$, at room temperature for $5 \mathrm{~min}$. Supernatants were discarded, and pellets were washed and centrifuged again at $670 \mathrm{x}$ g for $5 \mathrm{~min}$. Pellets were mixed with preservation solution (Hologic, Inc., Bedford, Massachusetts, USA). ThinPrep slides were made automatically by Thinprep 5000 (Product Insight Inc., Acton, MA, USA).

For cell block preparation, $200 \mathrm{ml}$ freshly collected pleural effusion was centrifuged at $670 \mathrm{x} g$ at room temperature for $5 \mathrm{~min}$. Pellets were fixed in $95 \%$ ethanol for $12 \mathrm{~h}$, embedded in paraffin, sectioned $(5 \mu \mathrm{m})$ and stained with hematoxylin and eosin (HE). The slides were visualized under a microscope (Nikon 80i; Nikon Corporation, Tokyo, Japan) and further observed by transmission electron microscopy (JEM-1400; JEOL Ltd., Tokyo, Japan).

Immunocytochemical stains. Immunocytochemical analysis was performed according to the manufacturer's instructions for EnVision (Maixin Biotech Co., Ltd., Fuzhou, China). Table I shows the details of the primary antibodies used. Paraffinized slides were placed into an oven at $60^{\circ} \mathrm{C}$ 
Table I. Primary antibodies used for immunohistochemistry and western blot analysis.

\begin{tabular}{|c|c|c|c|c|c|}
\hline \multirow[b]{2}{*}{ Antibody } & \multirow[b]{2}{*}{ Host } & \multicolumn{2}{|c|}{ Dilution } & \multirow[b]{2}{*}{ Source } & \multirow[b]{2}{*}{ Catalog no. } \\
\hline & & ICC & $\begin{array}{l}\text { Western } \\
\text { blot }\end{array}$ & & \\
\hline Cytokeratin 7 & Mouse monoclonal & $1: 100$ & - & Santa Cruz & sc-23876 \\
\hline TTF-1 & Rabbit polyclonal & $1: 100$ & - & Santa Cruz & sc- 13040 \\
\hline Vimentin & Mouse monoclonal & $1: 100$ & - & Santa Cruz & sc-53464 \\
\hline ERCC1 & Mouse monoclonal & $1: 50$ & $1: 500$ & Abcam & ab113941 \\
\hline TYMS & Rabbit polyclonal & $1: 50$ & $1: 500$ & Abcam & ab155795 \\
\hline TUBB3 & Rabbit monoclonal & $1: 50$ & $1: 500$ & Abcam & ab52901 \\
\hline RRM1 & Mouse monoclonal & $1: 50$ & $1: 500$ & Abcam & ab157250 \\
\hline $\begin{array}{l}\text { Nonmuscle } \\
\text { Myosin IIA }\end{array}$ & Rabbit polyclonal & $1: 50$ & $1: 500$ & Abcam & ab24762 \\
\hline Myoglobin & Mouse monoclonal & $1: 50$ & $1: 500$ & Abcam & ab47702 \\
\hline MyoD1 & Mouse monoclonal & $1: 50$ & $1: 500$ & Abcam & ab16148 \\
\hline
\end{tabular}

for $120 \mathrm{~min}$. Slides were then placed into xylene solution in order to remove paraffin and then into a graded ethanol series in order to remove the xylene. Slides were then rinsed in distilled water. Following deparaffinization and rehydration, the antigens were retrieved for $30 \mathrm{~min}$ at $100^{\circ} \mathrm{C}$ using Tris/Borate/EDTA cell-conditioning solution (Maixin Biotech Co., Ltd.). Primary antibodies were incubated separately for $60 \mathrm{~min}$ at room temperature, followed by incubation with either mouse or rabbit polymers (ZSGB-BIO Co., Ltd., Beijing, China) for $30 \mathrm{~min}$. Visualization was facilitated with enhanced diaminobenzidine chromagen (Maixin Biotech Co., Ltd.). Slides were counterstained with hematoxylin. Appropriate positive and negative controls were used to evaluate the human tissue samples, according to the manufacturer's instructions. Phosphate-buffered saline was used as the negative control.

Dark brown staining observed in either the cytoplasm or nuclei of cells was counted as a positive result. The average percentage of positive cells in five randomly-selected high power fields (x200) was calculated using a microscope (Nikon 80i, Nikon Corporation, Tokyo, Japan). Samples with $>5 \%$ positive cells were regarded as positive for that stain. The immunocytochemical stains were evaluated by three attending cytopathologists, and the results shown are an average of the scores obtained by these individuals.

Western blot analysis. Pleural effusions were collected and stored at $-70^{\circ} \mathrm{C}$ in a freezer. Total protein was extracted from cells in the effusion and the protein concentration was measured using a Bradford Protein Assay kit (Beyotime Institute of Biotechnology, Haimen, China) which is based on the absorbance of Coomassie Brilliant Blue G-250. The colorimetric absorbance of the samples was measured at 465-595 nm on an Enzyme Standard instrument (MK3; Thermo Fisher Scientific, Bedford, MA, USA). Following separation by electrophoresis on SDS-PAGE gels, protein was transferred to polyvinylidine fluoride membranes (Beyotime Institute of Biotechnology). Membranes were blocked with $5 \%$ non-fat milk for $2 \mathrm{~h}$ at room temperature and incubated with primary antibody (Table I) separately at $4^{\circ} \mathrm{C}$ overnight. Following washing in Tris-buffered saline with Tween-20 (Beyotime Institute of Biotechnology), membranes were incubated with goat anti-mouse polyclonal horseradish peroxidase-conjugated secondary antibodies (ZSGB-BIO. Co., Ltd.), at room temperature for $2 \mathrm{~h}$. Membranes were developed using Thermo chemiluminescent substrate kits (Thermo Fisher Scientific). $\beta$-actin was used as an internal control. Protein expression was quantified using a gel imaging analysis system (Systems BioImaging Lab, Santa Barbara, CA, USA).

Statistical analysis. Statistical data analysis was performed using SPSS17.0 statistical software (SPSS Inc., Chicago, IL, USA). The association between gene expression and clinical characteristics was analyzed using $\chi^{2}$ test or Fisher's exact test. The correlation between different chemoresistance factors was analyzed using Spearman's correlation coefficients. The Kaplan-Meier method and the Log-rank test were performed to analyze the relationship between patient survival and expression of the proteins investigated. Multivariate Cox regression analysis was used to analyze the correlation between age, gender and survival. $\mathrm{P}<0.05$ was considered to indicate a statistically significant difference.

\section{Results}

Pleural effusion cytology and immunocytochemistry. Diagnosis of metastatic lung adenocarcinoma was made cytologically from pleural fluid samples and confirmed by positive TTF-1 and CK7 staining in cell blocks (Fig. 1). 


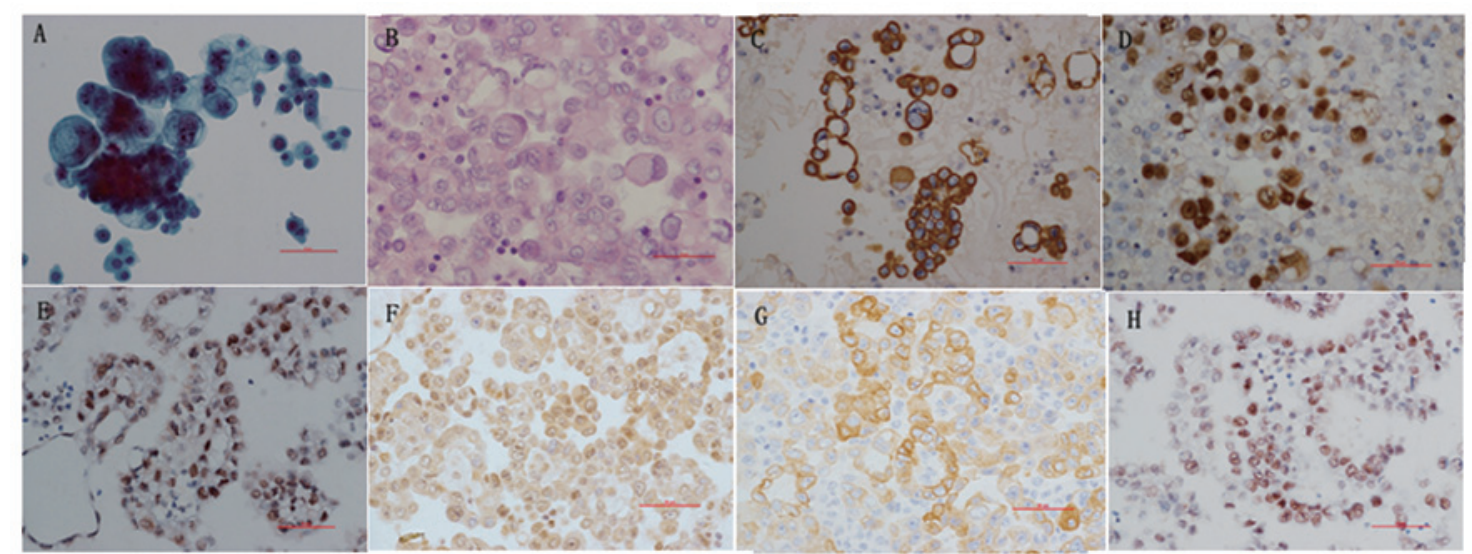

Figure 1. (A) ThinPrep cytology test of malignant pleural fluid (Papanicolaou, x400). (B) Cell block sections of cases of lung adenocarcinoma (hematoxylin and eosin staining; 400 ). (C) Immunocytochemistry tests CK7 (x400). (D) Thyroid transcription factor 1 was used to further detect the cells from malignant pleural fluid $(\mathrm{x} 400)$. (E) Expression of ERCC1 (x400) in lung adenocarcinoma cells from malignant effusion. (F) Expression of TYMS (x400) in lung adenocarcinoma cells from malignant effusion. (G) Expression of vimentin (x400) in lung adenocarcinoma cells from malignant effusion. (H) Expression of MyoD1 (x400) in lung adenocarcinoma cells from malignant effusion. CK7, cytokeratin 7; ERCC1, excision repair cross-complementing gene 1; TYMS, thymidylate synthase.

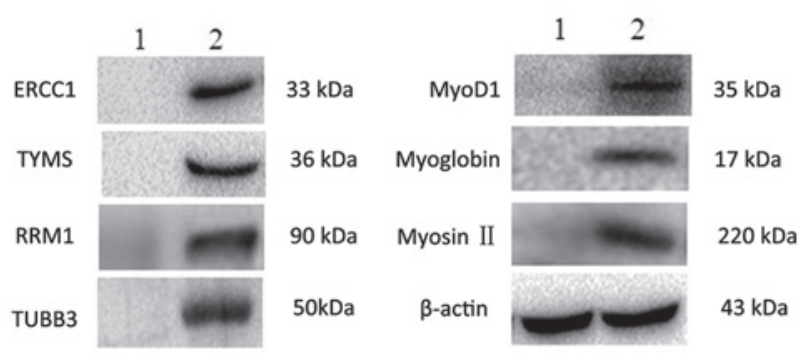

Figure 2. Expression of ERCC1, TYMS, RRM1, TUBB3, vimentin, myosin II, myoglobin and MyoD1 in cells from pleural fluid was analyzed by western blotting. Sample 1 contained cells from reactive inflammatory cell pleural effusions. Sample 2 contained cells from malignant pleural effusions in patients with a diagnosis of lung adenocarcinoma. $\beta$-actin was used as a control. ERCC1, excision repair cross-complementing gene 1; TYMS, thymidylate synthase; RRM1, ribonucleotide reductase M1; TUBB3, $\beta I I I-t u b u l i n$.

Protein expression of drug resistance-related factors. The expression of ERCC1, TYMS, RRM1, TUBB3 and vimentin was evaluated in the 116 samples from patients with metastatic lung adenocarcinoma (Fig. 1). The positive rate for ERCC1 was $37 \%$, with positive staining observed in the nuclei. The positive rate for TYMS was $36.2 \%$, with positive staining observed in the nuclei and cytoplasm. Positive rates for RRM1 and TUBB3 were 82.7 and $69.8 \%$, respectively, and positive staining was observed in the cytoplasm. The positive rate for vimentin was $83.6 \%$, with positive staining observed in the cytoplasm. In the subset of 50 patients with clinical follow-up who were treated with platinum-based chemotherapy, the expression of myosin II, myoglobin, and MyoD1 were also found to be upregulated (Fig. 1). The positive rate for myosin II was $48 \%$, with cytoplasmic staining observed. The positive rate for myoglobin was $40 \%$, with cytoplasmic staining observed. The positive rate for MyoD1 was 38\%, with nuclear staining observed.

The protein expression levels of ERCC1, TYMS, TUBB3, non-muscle myosin II, myoglobin and MyoD1 were also measured by western blot analysis. These proteins were not measurable in the inflammation-induced pleural fluids from the control group. However, they were all detectable in the pleural effusions from patients with metastatic lung adenocarcinoma (Fig. 2).

Correlation between drug resistance-related factor expression. In the 116 samples of pleural fluid from patients with metastatic lung adenocarcinomas there was a significant positive correlation between the expression of ERCC and TYMS ( $\mathrm{r}=0.54, \mathrm{P}=0.000$ ), between RRM1 and TUBB3 $(\mathrm{r}=0.45, \mathrm{P}=0.000)$, between ERCC 1 and TUBB3 $(\mathrm{r}=0.35$, $\mathrm{P}=0.000)$, between ERCC1 and RRM1 $(\mathrm{r}=0.26, \mathrm{P}=0.006)$, and between TYMS and TUBB3 $(r=0.22, P=0.017)$. The correlation between the expression of TYMS and RRM1 was not statistically significant, $(\mathrm{r}=0.06, \mathrm{P}=0.530$; Table II).

In the subset of 50 cases treated with platinum-based chemotherapy who received clinical follow-up, significantly positive correlations were identified between the expression of myoglobin and MyoD1 ( $\mathrm{r}=0.96, \mathrm{P}=0.000)$, between myosin II and MyoD1 ( $\mathrm{r}=0.81, \mathrm{P}=0.000)$, and between myosin II and myoglobin ( $\mathrm{r}=0.77, \mathrm{P}=0.000$; Table III). Expression of these three factors was also positively correlated with the expression of ERCC1, TYMS, RRM1 and TUBB3 (Table IV).

Association between protein expression and survival. Two out of 50 patients were lost to clinical follow-up. Kaplan-Meier survival analysis was performed in the remaining 48 cases. Patients with an undetectable expression of ERCC1, TYMS, and TUBB3 had a longer survival time compared with patients with a positive expression of these proteins (10.3 months and 7.4 months, $\mathrm{P}=0.000$; 10.6 months and 7.3 months, $\mathrm{P}=0.000$; and 11.6 months and 8.5 months, $\mathrm{P}=0.001$, respectively). Negative correlations were also observed between the expression of myosin II, myoglobin, and MyoD1 and survival. Positive expression was related to a worse prognosis (10.3 months compared with 7.6 months, $\mathrm{P}=0.008 ; 10.6$ months compared with 7.3 months, $\mathrm{P}=0.000$; and 10.6 months compared with 7.3 months, $\mathrm{P}=0.000$, respectively; Fig. 3). The expression of 
Table II. Correlation between expression of ERCC1, TYMS, RRM1 and TUBB3.

A, Presence of ERCC1 in relation to that of TYMS, TUBB3 and RRM1.

\begin{tabular}{|c|c|c|c|c|c|}
\hline & ERCC1 + & ERCC1 - & Total & $\mathrm{r}$ & P-value \\
\hline \multicolumn{6}{|c|}{ TYMS } \\
\hline+ & 30 & 12 & 42 & 0.54 & 0.000 \\
\hline- & 13 & 61 & 74 & & \\
\hline Total & 43 & 73 & 116 & & \\
\hline \multicolumn{6}{|c|}{ TUBB3 } \\
\hline+ & 39 & 42 & 81 & 0.35 & 0.000 \\
\hline- & 4 & 31 & 35 & & \\
\hline Total & 43 & 73 & 116 & & \\
\hline \multicolumn{6}{|c|}{ RRM1 } \\
\hline+ & 41 & 55 & 96 & 0.25 & 0.006 \\
\hline- & 2 & 18 & 20 & & \\
\hline Total & 43 & 73 & 116 & & \\
\hline
\end{tabular}

B, Presence of RRM1 in relation to that of TUBB3.

\begin{tabular}{lccccc}
\hline & RRM1 + & RRM1 & Total & r & P \\
\hline TUBB3 & & & & & 0.000 \\
+ & 76 & 5 & 81 & 0.45 & \\
- & 20 & 15 & 35 & & \\
Total & 96 & 20 & 116 & & \\
\hline
\end{tabular}

C, Presence of TYMS in relation to that of TUBB3 and RRM1.

\begin{tabular}{|c|c|c|c|c|c|}
\hline & TYMS + & TYMS - & Total & $\mathrm{r}$ & $\mathrm{P}$ \\
\hline \multicolumn{6}{|c|}{ TUBB3 } \\
\hline+ & 35 & 46 & 81 & 0.22 & 0.017 \\
\hline- & 7 & 28 & 35 & & \\
\hline Total & 42 & 74 & 116 & & \\
\hline \multicolumn{6}{|c|}{ RRM1 } \\
\hline+ & 36 & 60 & 96 & 0.06 & 0.530 \\
\hline- & 6 & 14 & 20 & & \\
\hline Total & 42 & 74 & 116 & & \\
\hline
\end{tabular}

ERCC1, excision repair cross-complementing gene 1; TYMS, thymidylate synthase; RRM1, ribobucleotide reductase M1, TUBB3, $\beta$ III-tubulin; $\mathrm{P}, \mathrm{P}$-value.

RRM1 showed no correlation with survival time $(\mathrm{P}=0.158)$. Survival was not associated with age or gender $(\mathrm{P}=0.517$ and $\mathrm{P}=0.592$, respectively).

\section{Discussion}

Chemotherapy is the treatment of choice for advanced or metastatic NSCLC. According to the recent non-small cell lung cancer treatment guideline from the National Comprehensive Cancer Network website (NCCN, Fort Washington,
PA, USA, 2011), cisplatin or carboplatin-based chemotherapy remains the first-line treatment for standard chemotherapy. The combination of a platinum-based drug with another drug, constitutes an effective treatment protocol (23). However, the development of drug resistance has become a significant problem. A change in the expression of a resistance protein may induce resistance towards one or more drugs. Platinum interacts with DNA to form Pt-DNA adducts, resulting in DNA cross-linking or chain cross-linking and the formation of an abnormal DNA double helix, thereby 
Table III. Correlation between expression of myosin II, myoglobin and MyoD1 (50 samples).

A, Presence of myosin II in relation to that of myoglobin anf MyoD1.

\begin{tabular}{lccccc}
\hline & Myosin II + & Myosin II & Total & r & P-value \\
\hline Myoglobin & & & & & 0.000 \\
+ & 19 & 1 & 20 & 0.77 & \\
- & 5 & 25 & 30 & & 0.000 \\
Total & 24 & 26 & 50 & 0.81 & \\
MyoD1 & 19 & 0 & 19 & & \\
+ & 5 & 26 & 31 & & \\
- & 24 & 26 & 50 & & \\
Total & & & & & \\
\hline
\end{tabular}

$\mathrm{B}$, Presence of myoglobin in relation to that of MyoD1.

\begin{tabular}{lccccc}
\hline & Myoglobin + & Myoglobin - & Total & r & P-value \\
\hline MyoD1 & & & & & 0.00 \\
+ & 19 & 0 & 19 & & \\
- & 1 & 30 & 31 & & \\
Total & 20 & 30 & 50 & & \\
\hline
\end{tabular}

resulting in apoptosis (24). ERCC1 clears cisplatin-induced DNA complexes and repairs the damaged DNA, leading to resistance to platinum-based therapies (25). Amongst patients with NSCLC treated with platinum-based chemotherapy, ERCC1-negative individuals exhibit a longer PFS than ERCC1-positive individuals $(26,27)$. The present study confirmed these observations, demonstrating a poor prognosis in patients with lung adenocarcinoma who expressed ERCC1 in tumor cells from pleural effusions. TYMS is a key enzyme in DNA synthesis, affecting the efficacy of drugs that target this process. Shimizu et al (28) showed that the level of expression of TYMS mRNA affected pemetrexed treatment efficacy. For patients with NSCLC receiving pemetrexed treatment, TYMS is a molecular marker, which predicts treatment effect. RRM1, like ERCC1 and TYMS, is important in DNA synthesis and repair. An association between its overexpression and gemcitabine resistance has previously been reported (29). In a meta-analysis, Gonga et al (30) showed that amongst patients with late-stage NSCLC receiving a gemcitabine chemotherapy regimen, a low or negative level of expression of RRM1 was associated with a higher rate of drug response, longer survival and time to progression. In healthy adult tissues, TUBB3 distribution is primarily limited to nervous system tissues. However, a number of studies have demonstrated TUBB3 expression in non-neurogenic solid tumors, such as breast cancer, ovarian cancer and colon cancer (31-35). The expression of TUBB3 in tumor cells may represent a 'dedifferentiation', indicating a greater malignant and metastatic capacity (36). High expression levels of TUBB3 in NSCLC, breast cancer, ovarian cancer and metastatic carcinoma have been reported to be associated with a lower sensitivity to vinblastine and paclitaxel, as well as lower survival rates (31-34,37). The present results indicate increased expression of ERCC1, RRM1, TYMS and TUBB3 in pleural effusions from a subpopulation of patients with metastatic lung adenocarcinoma. The expression of these factors appears to be correlated. The median survival of patients with detectable ERCC1, TYMS and TUBB3 expression was shorter than that of patients with negative expression of these proteins. The impact of RRM1 expression on survival was not found to be statistically significant.

Previous studies have shown that epithelial-mesenchymal transition (EMT) is important in tumor invasion, metastasis, the acquisition of stem cell-like characteristics and the development of drug resistance (38). Studies have demonstrated that gemcitabine-resistant pancreatic cancer cells undergo EMT via activation of the Notch signaling pathway. The transitioned cells exhibited decreased expression of E-cadherin and $\beta$-catentin, and increased expression of ZEB1, Snail and vimentin. They also displayed morphological alterations (39). An oxaliplatin-resistant colorectal cancer cell line developed a phenotype akin to that of mesenchymal cells, with long spindle-shaped cells, loss of polarity, loss of cell cohesiveness, increased metastasis and invasion, and changes in molecular markers (40). Similar changes have also been reported in paclitaxel-resistant ovarian cancer cell lines (41). Snail has been reported to enhance ERCC1 expression. It thus contributes to cisplatin resistance (42). The association between other drug-resistance genes and EMT is largely unknown. The present study measured myoglobin, myosin II and MyoD1 protein expression in 50 randomly-selected patients. Kocaefe et al (43) found that following MyoD1 transfection into umbilical cord blood mesenchymal stem cells, the stem cells displayed certain functional proteins characteristic of muscle cells, such as desmin, myosin and myoglobin. The results from the present study demonstrated the expression of 


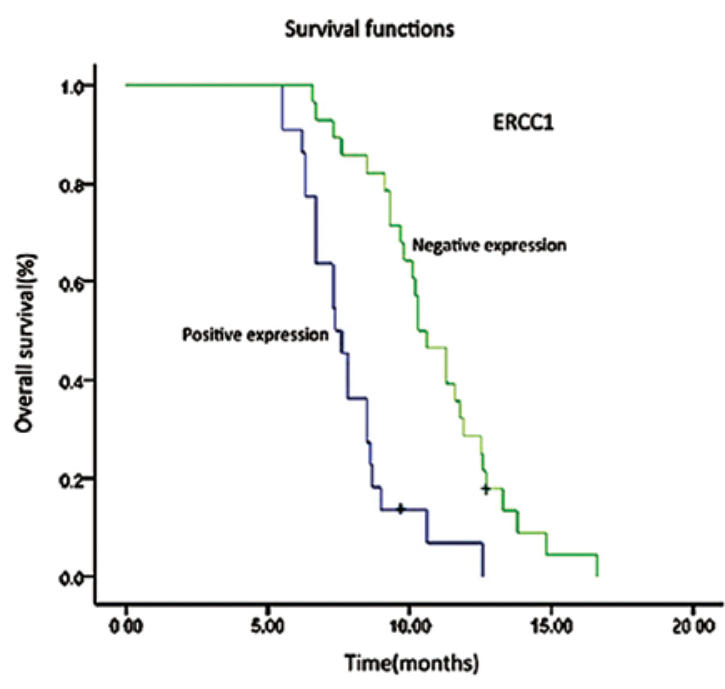

Sunival times according to ERCC1 expression (10.3 vs. 7.4 months, $P=0.000$ ).

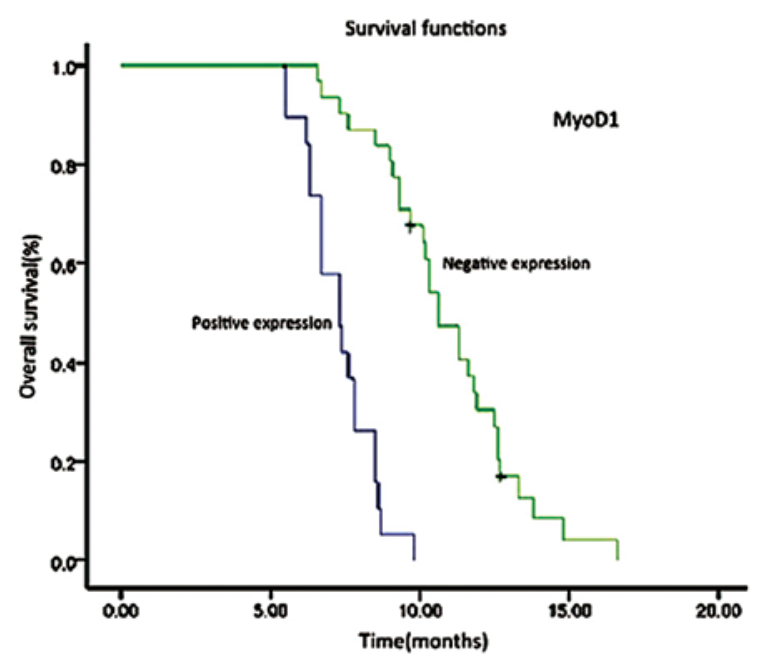

Survival times according to MyoD1 expression (10.6 vs. 7.3 months, $P$ $=0.000$ )

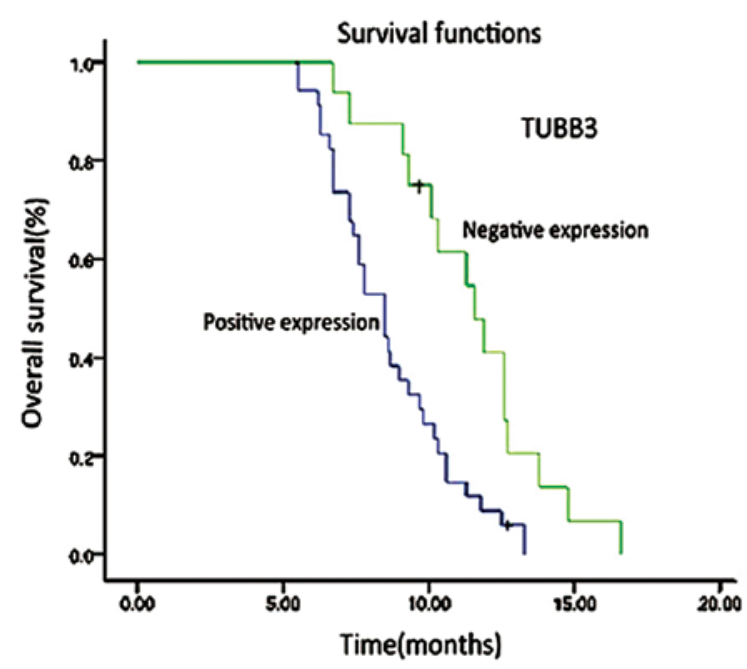

Survival times according to TUBB3 expression (11.6 vs. 8.5 months, $P=0.001$ ).

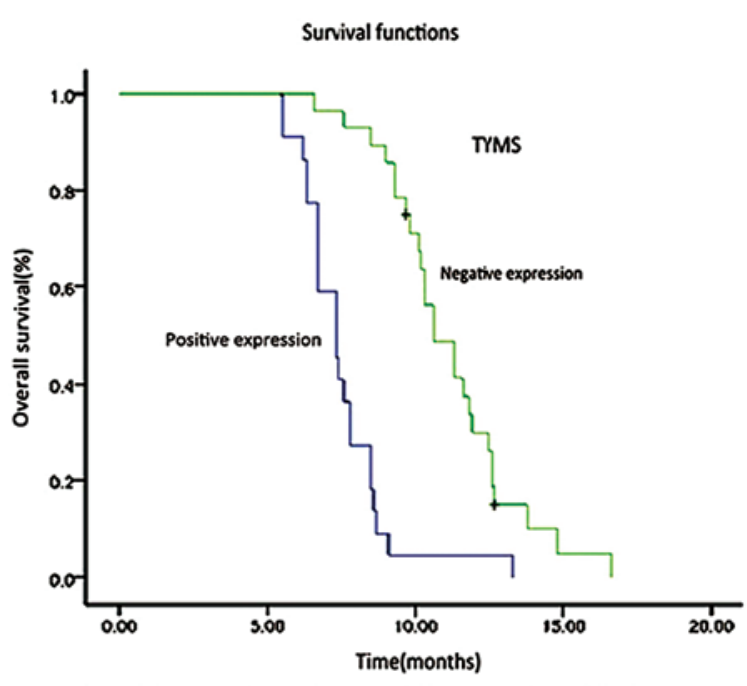

Sunvival times according to TrMS expression (10.6 vs. 73 months, $P=0.000$ )

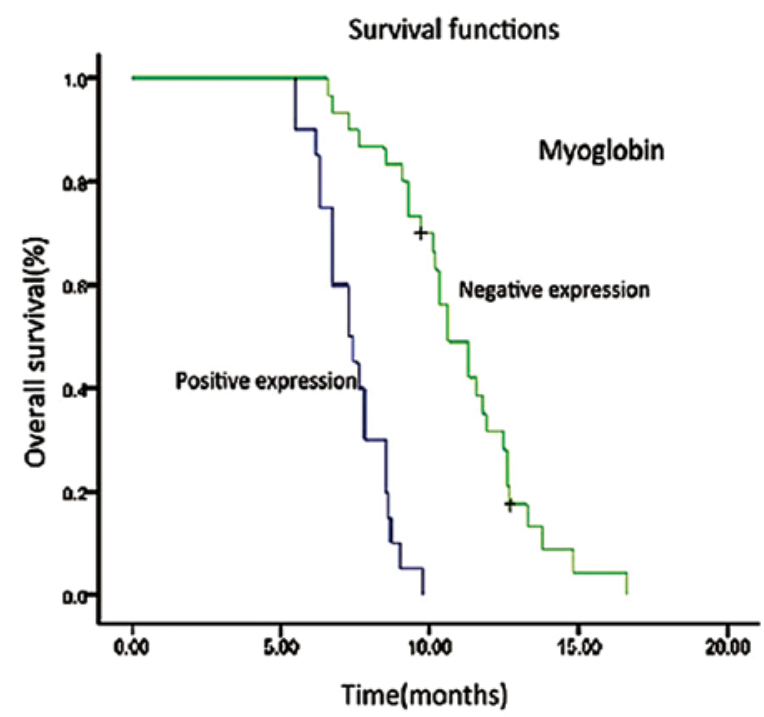

Survival times according to Myoglobin expression (10.6 vs. 7.3 months, $P=0.000$ ).

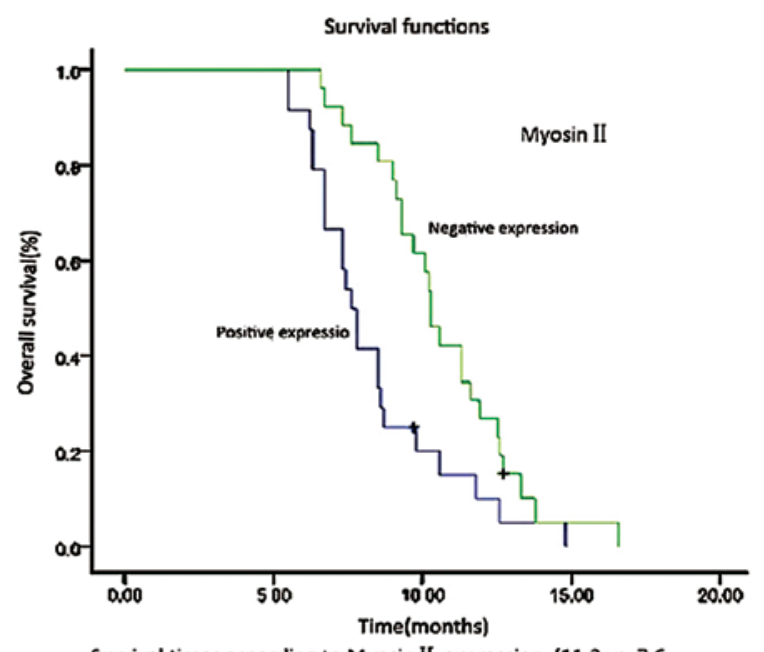

Survival times according to Myosin II expression (11.3 vs. 7.6 months, $P=0.008$ ).

Figure 3. Kaplan-Meier analysis of the correlation between ERCC1, TYMS, TUBB3, Myosin II, Myoglobin, MyoD1 expression and patient survival. ERCC1, excision repair cross-complementing gene 1; TYMS, thymidylate synthase, TUBB3, $\beta$ III-tubulin. 
Table IV. Correlation between expression of seven proteins (50 Samples).

A, Presence of myosin in relation to that of ERCC1, TYMS, TUBB3 and RRM1.

\begin{tabular}{|c|c|c|c|c|}
\hline & Myosin + & Myosin - & $\mathrm{r}$ & P-value \\
\hline \multicolumn{5}{|c|}{ ERCC1 } \\
\hline+ & 20 & 1 & 0.8 & 0.000 \\
\hline - & 4 & 25 & & \\
\hline \multicolumn{5}{|c|}{ TYMS } \\
\hline+ & 18 & 4 & 0.6 & 0.000 \\
\hline- & 6 & 22 & & \\
\hline \multicolumn{5}{|c|}{ TUBB3 } \\
\hline+ & 21 & 13 & 0.4 & 0.004 \\
\hline- & 3 & 13 & & \\
\hline \multicolumn{5}{|c|}{ RRM1 } \\
\hline+ & 24 & 17 & 0.45 & 0.001 \\
\hline- & 0 & 9 & & \\
\hline
\end{tabular}

B, Presence of myoglobin in relation to that of ERCC1, TYMS, TUBB3 and RRM1.

\begin{tabular}{|c|c|c|c|c|}
\hline & Myglobin + & Myoglobin - & $\mathrm{r}$ & P-value \\
\hline \multicolumn{5}{|c|}{ ERCC1 } \\
\hline+ & 18 & 3 & 0.79 & 0.000 \\
\hline- & 2 & 27 & & \\
\hline \multicolumn{5}{|c|}{ TYMS } \\
\hline+ & 18 & 4 & 0.76 & 0.000 \\
\hline- & 2 & 26 & & \\
\hline \multicolumn{5}{|c|}{ TUBB3 } \\
\hline+ & 20 & 14 & 0.56 & 0.000 \\
\hline- & 0 & 16 & & \\
\hline \multicolumn{5}{|c|}{ RRM1 } \\
\hline+ & 20 & 21 & 0.38 & 0.006 \\
\hline- & 0 & 9 & & \\
\hline
\end{tabular}

C, Presence of MyoD1 in relation to that of ERCC1, TYMS, TUBB3 and RRM1.

\begin{tabular}{|c|c|c|c|c|}
\hline & MyoD1 + & MyoD1 - & $\mathrm{r}$ & $\mathrm{P}$-value \\
\hline \multicolumn{5}{|c|}{ ERCC1 } \\
\hline+ & 17 & 4 & 0.75 & 0.000 \\
\hline- & 2 & 27 & & \\
\hline \multicolumn{5}{|c|}{ TYMS } \\
\hline+ & 18 & 4 & 0.80 & 0.000 \\
\hline- & 1 & 27 & & \\
\hline \multicolumn{5}{|c|}{ TUBB3 } \\
\hline+ & 19 & 15 & 0.54 & 0.000 \\
\hline- & 0 & 16 & & \\
\hline \multicolumn{5}{|c|}{ RRM1 } \\
\hline+ & 19 & 22 & 0.37 & 0.009 \\
\hline- & 0 & 9 & & \\
\hline
\end{tabular}

ERCC1, excision repair cross-complementing gene 1; TYMS, thymidylate synthase; TUBB3, $\beta$ III-tubulin; RRM1, ribonucleotide reductase M1. 
myoglobin, myosin II and MyoD1 in lung adenocarcinoma cells obtained from pleural effusions. The expression of each of these markers was positively correlated. To the best of our knowledge, MyoD1 expression in lung cancer has not been previously reported in the literature. Giarnieri et al (44) found that when lung adenocarcinoma in pleural effusions underwent EMT, the tumor cells lost expression of E-cadherin and exhibited enhanced expression of $\mathrm{N}$-cadherin. In the current study, cancer cells expressed not only the epithelial marker, CK7, but also the mesenchymal marker, vimentin, in $83.6 \%$ of cases. One explanation for the increased expression of myglobin, myosin II and MyoD1, is that these proteins are by-products of adenocarcinoma cells undergoing the EMT process. Tumor cells may acquire certain mesenchymal characteristics and upregulate the expression of MyoD1, followed by the expression of myoglobin and myosin II. Furthermore, increased expression of myosin II may also be a response to an increase in cytoskeletal activity. The cytoskeleton is important in maintaining cell morphology, intracellular transportation and cellular motility. The present results showed increased cytoskeletal components, TUBB3 and myosin II, in pleural fluid samples from certain patients with metastatic lung adenocarcinoma. These are key components of microtubules and actin filaments, respectively. Such changes may enhance the metastatic capability of late-stage tumor cells. In the present study TCT and HE slides were produced, and visualized under an electron microscope. Increased myosin II protein expression levels were shown to be associated with fibrous components in the cytoplasm, with a reticular pattern. The fibrous net may form a mechanical barrier against chemotherapy drugs, thereby facilitating the development of drug resistance.

The present study also showed that expression of the myoglobin, myosin II and MyoD1 proteins was positively correlated with the expression of four known drug-resistance genes. Snail, a key regulator of the EMT process, is known to increase ERCC1 expression. The current results further suggest the importance of EMT in the malignant transformation of tumor cells. Oleksiewicz et al (45) observed myoglobin expression in primary lung adenocarcinoma. Individuals with a higher expression level had a shorter average survival time, which may have been associated with the increased anti-hypoxia capacity of the tumors. Xiong et al (46) found that in patients with early-stage bladder cancer, high myosin II expression was associated with a lower five-year survival rate, as well as an increase in lymph node metastasis. The results from the present study also illustrated that the average survival time of myoglobin, myosin II and MyoD1-positive patients was shorter than that of negative patients. The exact mechanisms underlying this reduction in survival time are unknown. We hypothesize that this expression pattern is a common phenotype in all highly-malignant tumor cells and increases tumor cell resistance to hypoxia, nutritional deficiencies and chemotherapy treatment. This phenotype also enhances the invasion and metastasis of tumor cells, which gain certain cancer stem cell-like properties.

The enhanced protein expression of drug resistance genes increases DNA synthesis in tumor cells, accelerates tumor proliferation, promotes tumor cell invasion and metastasis, and induces resistance to drugs targeting these molecules. Sensitivity to standard chemotherapy varies significantly among individuals, even those with the same pathological type and stage of tumors. In recent years the concept of individualized medicine has been proposed. The current data showed drug resistance protein expression in samples of pleural fluid from subsets of patients with metastatic lung adenocarcinoma. The expression of these proteins was correlated with one another and the overall survival in patients expressing drug resistance factors was lower than that in patients in whom these factors were undetectable. The collection of pleural fluid is a technically simple method for obtaining tumor cells. Immunocytochemical analysis of drug resistance gene protein expression is a rapid and convenient approach to analyze the expression of these drug resistance genes. The results of immunocytochemical analyses may provide a reliable basis for the choice of individualized chemotherapy protocols, thus improving prognosis and potentially reducing treatment costs.

\section{References}

1. Siegel R, Naishadham D and Jemal A: Cancer statistics, 2013. CA Cancer J Clin 63: 11-30, 2013.

2. Marshall HM, Leong SC, Bowman RV, et al: The science behind the 7th edition Tumour, Node, Metastasis staging system for lung cancer. Respirology 17: 247-260, 2012.

3. Morgensztern D, Waqar S, Subramanian J, et al: Prognostic impact of malignant pleural effusion at presentation in patients with metastatic non-small-cell lung cancer. J Thorac Oncol 7: 1485-1489, 2012

4. Zahreddine $\mathrm{H}$ and Borden LB: Mechanisms and insights into drug resistance in cancer. Front Pharmacol 4: 28, 2013.

5. Suh DH, Kim MK, Kim HS, Chung HH and Song YS: Epigenetic therapies as a promising strategy for overcoming chemoresistance in epithelial ovarian cancer. J Cancer Prev 18: 227-234, 2013.

6. Zhang L, Yang $\mathrm{H}$ and $\mathrm{Xu} \mathrm{J}$ : Gene expression significance in personalized medicine of non-small-cell lung cancer and gene expression analyzing platforms. Curr Drug Metab 12: 455-459, 2011.

7. Sancar A and Reardon JT: Nucleotide excision repair in E. coli and man. Adv Protein Chem 69: 43-71, 2004.

8. Van Den Broeck A, Nissou D, Brambilla E, et al: Activation of a Tip60/E2F1/ERCC1 network in human lung adenocarcinoma cells exposed to cisplatin. Carcinogenesis 33: 320-325, 2012.

9. Olaussen KA, Dunant A, Fouret P, et al: DNA repair by ERCC1 in non-small-cell lung cancer and cisplatin-based adjuvant chemotherapy. N Engl J Med 355: 983-991, 2006.

10. Xianjun F, Xiu-guang Q, Li Z, et al: ERCC1 and BRCA1 mRNA expression predicts the clinical outcome of non-small cell lung cancer receiving platinum-based chemotherapy. Pak J Med Sci 30: 488-492, 2014.

11. Gangjee A, Yu J, McGuire JJ, et al: Design, synthesis, and X-ray crystal structure of a potent dual inhibitor of thymidylate synthase and dihydrofolate reductase as an antitumor agent. J Med Chem 43: 3837-3851, 2000.

12. Sigmond J, Backus HH, Wouters D, et al: Induction of resistance to the multitargeted antifolate Pemetrexed (ALIMTA) in WiDr human colon cancer cells is associated with thymidylate synthase overexpression. Biochem Pharmacol 66: 431-438, 2003.

13. Wang T, Chuan Pan C, Rui Yu J, et al: Association between TYMS expression and efficacy of pemetrexed-based chemotherapy in advanced non-small cell lung cancer: a meta-analysis. PLoS one 8: e74284, 2013.

14. Rosell R, Cobo M, Isla D, et al: Pharmacogenomics and gemcitabine. Ann Oncol 17 (Suppl 5): 13-16, 2006.

15. Vilmar AC, Santoni-Rugiu E and Sorensen JB: Predictive impact of RRM1 protein expression on vinorelbine efficacy in NSCLC patients randomly assigned in a chemotherapy phase III trial. Ann Oncol 24: 309-314, 2013.

16. Katsetos CD, Herman MM and Mörk SJ: Class III beta-tubulin in human development and cancer. Cell Motil Cytoskeleton 55: 77-96, 2003.

17. McCarroll JA, Gan PP, Liu M and Kavallaris M: betaIII-tubulin is a multifunctional protein involved in drug sensitivity and tumorigenesis in non-small cell lung cancer. Cancer Res 70: 4995-5003, 2010. 
18. Kim JH and Adelstein RS: LPA(1)-induced migration requires nonmuscle myosin II light chain phosphorylation in breast cancer cells. J Cell Physiol 226: 2881-2893, 2011.

19. Merx MW, Flögel U, Stumpe T, et al: Myoglobin facilitates oxygen diffusion. FASEB J 15: 1077-1079, 2001

20. Flonta SM, Arena S, Pisacane A, et al: Expression and functional regulation of myoglobin in epithelial cancers. Am J Pathol 175: 201-206, 2009.

21. Jin W, Liu Y, Chen L, et al: Involvement of MyoD and c-myb in regulation of basal and estrogen-induced transcription activity of the BRCA1 gene. Breast Cancer Res Treat 125: 699-713, 2011.

22. Soleimani VD, Yin H, Jahani-Asl A, et al: Snail regulates MyoD binding-site occupancy to direct enhancer switching and differentiation-specific transcription in myogenesis. Mol Cell 47: 457-468, 2012

23. Polo V and Besse B: Maintenance strategies in stage IV non-small-cell lung cancer (NSCLC): in which patients, with which drugs? Ann Oncol 25: 1283-1293, 2014.

24. Hildebrandt MA, Gu J and $\mathrm{Wu} X$ : Pharmacogenomics of platinum-based chemotherapy in NSCLC. Expert Opin Drug Metab Toxicol 5: 745-755, 2009.

25. Ota S, Ishii G, Goto K, et al: Immunohistochemical expression of BCRP and ERCC1 in biopsy specimen predicts survival in advanced non-small-cell lung cancer treated with cisplatin-based chemotherapy. Lung Cancer 64: 98-104, 2009.

26. Das M, Riess JW, Frankel P, et al: ERCC1 expression in circulating tumor cells (CTCs) using a novel detection platform correlates with progression-free survival (PFS) in patients with metastatic non-small-cell lung cancer (NSCLC) receiving platinum chemotherapy. Lung Cancer 77: 421-426, 2012.

27. Gao Z, Han B, Shen J, et al: ERCC1 protein as a guide for individualized therapy of late-stage advanced non-small cell lung cancer. Exp Ther Med 2: 811-815, 2011.

28. Shimizu T, Nakanishi Y, Nakagawa Y, et al: Association between expression of thymidylate synthase, dihydrofolate reductase, and glycinamide ribonucleotide formyltransferase and efficacy of pemetrexed in advanced non-small cell lung cancer. Anticancer Res 32: 4589-4596, 2012.

29. Dong X, Hao Y, Wei Y, et al: Response to first-line chemotherapy in patients with non-small cell lung cancer according to RRM1 expression. PLoS One 9: e92320, 2014.

30. Gong W, Zhang X, Wu J, et al: RRM1 expression and clinical outcome of gemcitabine-containing chemotherapy for advanced non-small-cell lung cancer: a meta-analysis. Lung Cancer 75 : 374-380, 2012.

31. Dumontet C, Isaac S, Souquet PJ, et al: Expression of class III beta tubulin in non-small cell lung cancer is correlated with resistance to taxane chemotherapy. Bull Cancer 92: E25-E30, 2005.

32. Sève $P$, Isaac $S$, Trédan $O$, et al: Expression of class III $\beta$-tubulin is predictive of patient outcome in patients with non-small cell lung cancer receiving vinorelbine-based chemotherapy. Clin Cancer Res 11: 5481-5486, 2005.
33. Ferrandina G, Zannoni GF, Martinelli E, et al: Class III beta-tubulin overexpression is a marker of poor clinical outcome in advanced ovarian cancer patients. Clin Cancer Res 12: 2774-2779, 2006

34. Tommasi S, Mangia A, Lacalamita R, et al: Cytoskeleton and paclitaxel sensitivity in breast cancer: the role of beta-tubulins. Int J Cancer 120: 2078-2085, 2007.

35. Portyanko A, Kovalev P, Gorgun J and Cherstvoy E: beta(III)-tubulin at the invasive margin of colorectal cancer: possible link to invasion. Virchows Arch 454: 541-548, 2009.

36. Katsetos CD and Dráber P: Tubulins as therapeutic targets in cancer: from bench to bedside. Curr Pharm Des 18: 2778-2792, 2012.

37. Maus MK, Mack PC, Astrow SH, et al: Histology-related associations of ERCC1, RRM1, and TS biomarkers in patients with non small-cell lung cancer: implications for therapy. J Thoracic Oncol 8: 582-586, 2013.

38. Polyak K and Weinberg RA: Transitions between epithelial and mesenchymal states: acquisition of malignant and stem cell traits. Nat Rev Cancer 9: 265-273, 2009.

39. Wang Z, Li Y, Kong D, et al: Acquisition of epithelial-mesenchymal transition phenotype of gemcitabine-resistant pancreatic cancer cells is linked with activation of the notch signaling pathway. Cancer Res 69: 2400-2407, 2009.

40. Yang AD, Fan F, Camp ER, et al: Chronic oxaliplatin resistance induces epithelial-to-mesenchymal transition in colorectal cancer cell lines. Clin Cancer Res 12: 4147-4153, 2006.

41. Kajiyama H, Shibata K, Terauchi M, et al: Chemoresistance to paclitaxel induces epithelial-mesenchymal transition and enhances metastatic potential for epithelial ovarian carcinoma cells. Int J Oncol 31: 277-283, 2007.

42. Hsu DS, Lan HY, Huang CH, et al: Regulation of excision repair cross-complementation group 1 by Snail contributes to cisplatin resistance in head and neck cancer. Clin Cancer Res 16: 4561-4571, 2010

43. Kocaefe C, Balci D, Hayta BB and Can A: Reprogramming of human umbilical cord stromal mesenchymal stem cells for myogenic differentiation and muscle repair. Stem Cell Rev 6: $512-522,2010$

44. Giarnieri E, De Vitis C, Noto A, et al: EMT markers in lung adenocarcinoma pleural effusion spheroid cells. J Cell Physiol 228: 1720-1726, 2013.

45. Oleksiewicz U, Daskoulidou N, Liloglou T et al: Neuroglobin and myoglobin in non-small cell lung cancer: expression, regulation and prognosis. Lung Cancer 74: 411-418, 2011.

46. Xiong D, Ye YL, Chen MK, et al: Non-muscle myosin II is an independent predictor of overall survival for cystectomy candidates with early-stage bladder cancer. Oncol Rep 28 : $1625-1632,2012$ 